\title{
Electrical Isolation Preserved by Plasma Focused Ion Beam TEM Sample Preparation and Verified with STEM SEEBIC Imaging
}

Matthew Mecklenburg ${ }^{1}$, Fred Shaapur ${ }^{2}$, William Hubbard ${ }^{3}$, Brian Zutter ${ }^{4}$ and B. C. Regan ${ }^{4}$

${ }^{1}$ University of Southern California, Los Angeles, California, United States, ${ }^{2}$ FabMetrix, Inc., Scottsdale, Arizona, United States, ${ }^{3}$ NanoElectroning Imaging, Inc. (NEI), Los Angeles, California, United States,

${ }^{4}$ Department of Physics \& Astronomy, University of California, Los Angeles, Los Angeles, California, United States

TEM is a central tool for the study of microelectronics, with thin $(\sim 100 \mathrm{~nm})$ samples most commonly produced from components via focused ion beam (FIB) extraction of thin cross sections [1]. Scanning TEM electron beam-induced current (STEM EBIC) imaging provides electronic imaging contrast to complement the physical and chemical contrast given by more standard TEM techniques. However, the inevitable implantation of ions into the material alters the local electrical conductivity dramatically, especially in silicon where gallium acts as a dopant. The damage and contamination associated with $\mathrm{Ga}^{+}$ FIB sample preparation often causes significant leakage, destroying the device's electronic structure and function. Plasma FIBs with a xenon source offer an attractive alternative: the noble gas is non-conductive and does not dope the sample, readily diffusing from an implantation site out of the sample.

In STEM EBIC, current is measured on the sample via a transimpedance amplifier (TIA) and plotted pixel-by-pixel as the electron beam scans the sample. Different EBIC modes are accessible depending on the device, circuit, and TIA sensitivity [2,3]. A recently developed technique based on the secondary electron EBIC (SEEBIC) [2] provides access to information about the electrical properties, including the work function [2,4], resistance, electric field, and potential [3] in devices at high resolution [5]. SEEBIC has been demonstrated on off-the-shelf components prepared with standard $\mathrm{Ga}^{+} \mathrm{FIB}$ [3], however production of such samples is impractically slow and requires painstaking effort. Here we demonstrate low-noise STEM EBIC on samples prepared via xenon plasma FIB (PFIB). Differential EBIC connectivity maps show that naturally insulating layers (e.g. silicon nitride) remain insulating after the PFIB preparation.

A cross section of a FD10D photodiode (Thorlabs, Inc.) with $\sim 300 \times 2800 \times 100 \mathrm{~nm}^{3}$ silicon nitride cap was made and connected to two electrical contacts on a specialized TEM lift-out grid [6]. Figure 1 shows a schematic of the experiment. The cross section was cut such that the silicon nitride layer isolates two otherwise conducting sides of the device. The impedance of the sample, as determined by a current-voltage measurement, was $2.7 \mathrm{G} \Omega$. The EBIC in Figure 2 were acquired with the TIA connected to each side of the nitride, with the other side grounded in each case. On both sides of the nitride barrier, beam-induced hole current reaches ground through the more well-connected side of the device. In each image, the EBIC appears bright when the beam is incident on the side of the device electrically connected to the TIA. It is clearly seen which paths the hole current can use to reach the TIA, and which paths are impeded [7]. 


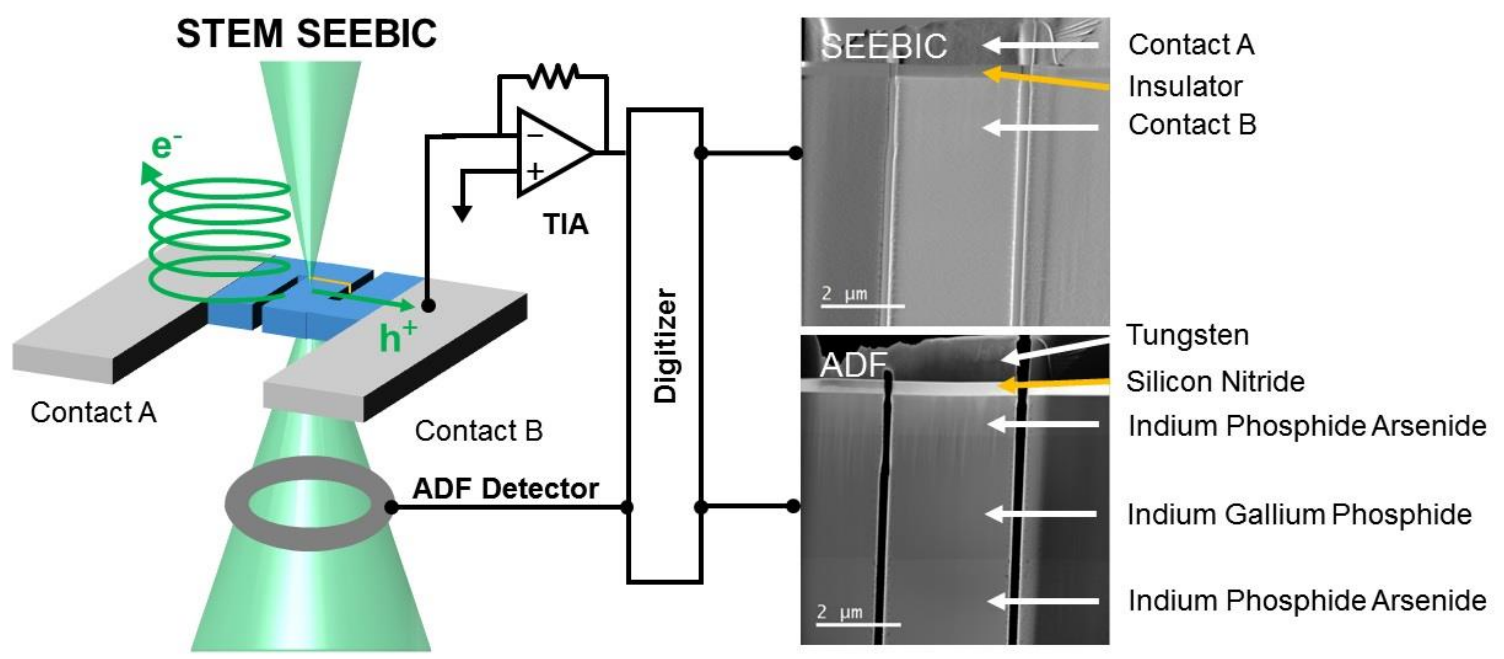

Figure 1. A schematic of the device and FIB cuts to provide electrical isolation. The device is shown connected to Contact B. During the experiment the connection was switched between Contact A and B. The layers in the photodiode are also shown.

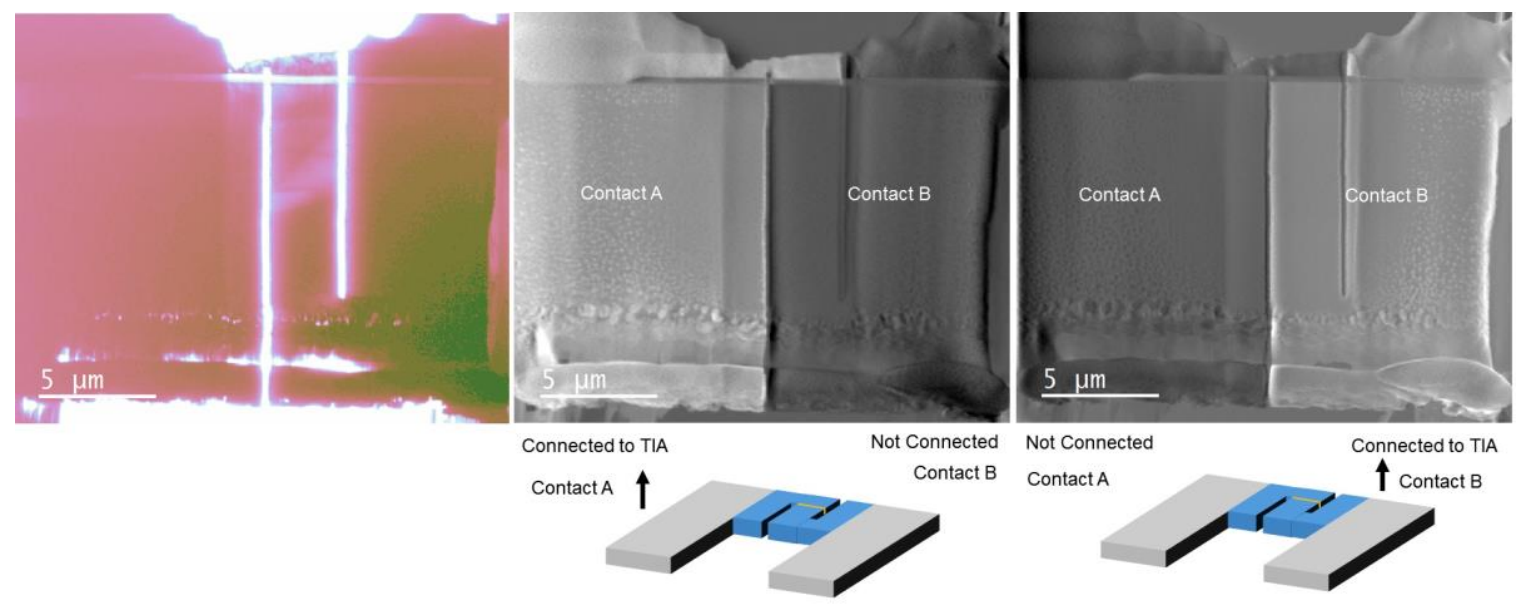

Figure 2. A low magnification BF image of the device shown with a CubeHelix color look up table to provide sufficient observable contrast. The SEEBIC images are shown to the right. The middle image shows the results of connection to Contact $A$ and the right image shows the opposing results when connected to Contact B. Brighter grayscale means a greater hole current in the SEEBIC images.

\section{References}

[1] J Mayer et al., MRS BULLETIN 32 (2007), p. 400.

[2] WA Hubbard et al., Physical Review Applied 10 (2018), p. 044066.

[3] W A Hubbard et al., Applied Physics Letters 115 (2019), p. 133502.

[4] M Mecklenburg et al., Microscopy and Microanalysis 25 (S2) (2019), p. 2354-2355.

[5] M Mecklenburg et al., Ultramicroscopy 207 (2019), p. 112852.

[6] M Mecklenburg et al., Microscopy and Microanalysis 19 (S2) (2013), p. 458-459.

[7] Data presented here were acquired at the Core Center of Excellence in Nano Imaging (CNI) at the University of Southern California. This work was supported by NSF STC award DMR-1548924 (STROBE), by NSF award DMR-1611036, and by the UCLA PSEIF. 